\title{
Effect of Protonation on the electronic properties of DNA base pairs: Applications for molecular electronics
}

\author{
Sairam S. Mallajosyula and Swapan K Pati \\ Theoretical Sciences Unit, Jawaharlal Nehru Center for Advanced \\ Scientific Research, Jakkur Campus, Bangalore 560 064, India.
}

(Dated: July 23, 2021)

\begin{abstract}
Protonation of DNA basepairs is a reversible phenomenon which can be controlled by tuning the $\mathrm{pH}$ of the system. Under mild acidic conditions, the hydrogen bonding pattern of the DNA basepairs undergoes a change. We study the effect of protonation on the electronic properties of the DNA basepairs to probe for possible molecular electronics applications. We find that, under mild acidic $\mathrm{pH}$ conditions, the A:T basepair shows excellent rectification behaviour which is, however, absent in the G:C basepair. The mechanism of rectification has been discussed using a simple chemical potential model. We also consider the non-canonical A:A basepair and find that it can be used as efficient $\mathrm{pH}$ dependent molecular switch. The switching action in A:A basepair is explained in the light of $\pi-\pi$ interactions which lead to efficient delocalization over the entire basepair.
\end{abstract}

The research on electron transport through molecules is gaining a lot of attention in recent years due to their potential applications in a large class of nanoscale technologies [1]. With advances in nanotechnology, there has been an active effort to use biomolecules for molecular electronic applications. This has been especially true for DNA based electronics owing to the unique geometry and stability of DNA which is built up on weak intermolecular $\pi$-stacking and specific hydrogen bonding (Hbonding) interactions between Adenine:Thymine (A:T) and Guanine:Cytosine (G:C) basepairs 2 .

So far, the studies on DNA conductivity have been mostly restricted to understanding the effect of $\pi$ - $\pi$ interactions between the DNA bases. This is due to the fact that experimental work on DNA conductance is carried out with DNA strands and the measurements are always between end to end of the DNA strand, or, in other words, about the helical axis of the DNA strand 3 . The importance of $\mathrm{H}$-Bonding in these experiments have been restricted to the formation of the DNA double helix, which stems from the specific basepairing pattern among the DNA bases.

In this work, we have analyzed the role played by the $\mathrm{H}$-bonding between the DNA bases under the influence of mild acidic conditions. It is known that the protonation of the DNA basepairs under mild acidic conditions is a reversible phenomenon and that it disrupts the H-Bonding profile between the DNA bases 4. We find that, this protonation under mild conditions changes the electronic properties of the DNA basepairs significantly and the effect being different for different basepairs. The effects of $\mathrm{pH}$ dependent reversible protonation-deprotonation on the electronic properties of a molecular system have been recently studied experimentally for di-block molecular diodes, where the inversion of the rectifying effect was found on selective protonation and deprotonation [5].

We have used the SIESTA and Gaussian 03 codes for our first principles DFT simulations [6, 7]. Within SIESTA, we have used the generalized gradient approx- imation for the exchange-correlation energy functional in the version of Perdew, Burke, and Ernzerhof $[8]$. A double- $\zeta$ basis set with the polarization orbitals has been included for all the atoms 9 . To model the gold electrodes, we placed two 27 atoms gold clusters at either end of the DNA basepairs. The coordinates of the gold clusters were obtained by relaxing two $\mathrm{Au}(111)$ surfaces separated by $30 \AA$, in a periodically repeated cell. Each surface was comprised of three $\mathrm{Au}(111)$ planes in a $2 \times 2$ surface unit cell along the [111] direction. The clusters thus derived from the relaxed surface calculations were then attached to the DNA basepairs via a thiol linker $\left(-\mathrm{CH}_{2} \mathrm{~S}\right)$. The Sulphur head group of the thiol linker was aligned on the fcc hollow site of the $\mathrm{Au}(111)$ surface using S-Au distance of $2.51 \AA$, previously reported in literature [10]. For the subsequent SIESTA calculations, the initial geometries of the basepairs were generated by Gaussian 03 calculations. Within Gaussian 03, we have used the polarizable continuum model with water dielectric to model the solvation effects. The large dielectric continuum of water reduces the electrostatic repulsions and stabilizes the formal positive charges introduced due to protonation. We have used the LANL2DZ basis set for obtaining the structures of the basepairs constrained between the two gold electrodes, wherein the gold electrodes are modeled as an equilateral triangle with an $\mathrm{Au}-\mathrm{Au}$ distance of $2.9 \AA$ which was obtained from the relaxed gold surfaces and which matches well with the previously reported experimental results [11]. All the calculations in SIESTA for the DNA basepairs attached to the gold clusters have been performed in a $30 \times 30 \times 60 \AA^{3}$ supercell, which is large enough for interactions between the neighboring fragments to be negligible. To model the reversible denaturation of the DNA basepairs, which can be controlled by $\mathrm{pH}$ variation, we have considered only the protonation of the amino nitrogen's, which can be easily achieved under mild acidic conditions [12].

Using the above described methodology, we have studied three different basepairs, namely, A:T (Ade- 

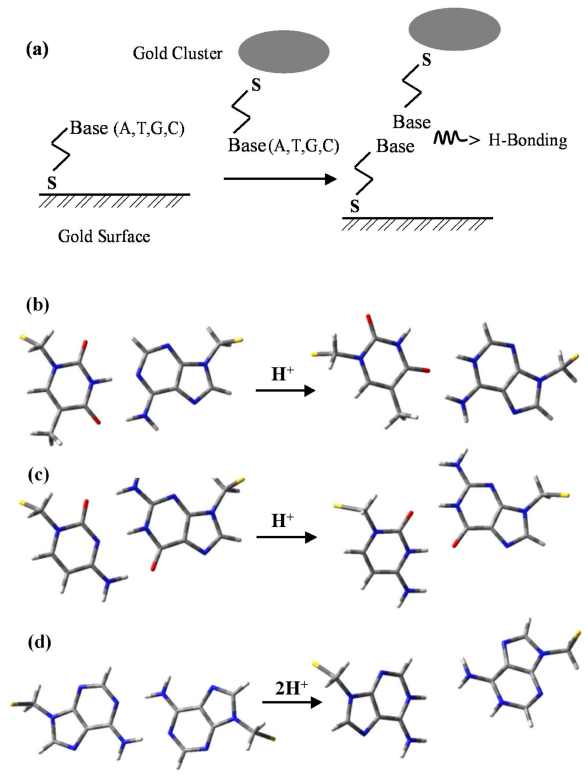

FIG. 1: (color online) (a) Probable scheme for experimental realization. Optimized geometries of the DNA basepairs: (b)

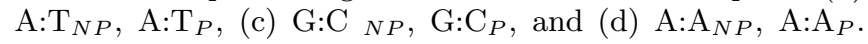
The Gold clusters have been omitted for clarity.

nine:Thymine), G:C (Guanine:Cytosine), and A:A (Adenine: Adenine). The first two are the naturally occurring watson-crick basepairs where the number of $\mathrm{H}$-bonds between the bases are two for A:T and three for G:C. As a first observation we note that on protonation both Adenine :Thymine (A:T) and Guanine:Cytosine (G:C) basepairs lose the N-H...N H-bond, however, they still retain the N-H...O H-bond. Thus, for a complete loss of Hbonding under mild acidic conditions, we consider the non-canonical Adenine:Adenine (A:A) basepair, which loses both the N-H...N H-bonds on protonation. Here, we would like to point out that the systems studied by us can be realized by experimental synthesis. We give a probable scheme for the experimental realization in Fig.1(a) based on the pioneering work of Mirkin et al. and Alivisatos et al. [13. The first step in the experimental method involves attaching DNA bases capped with thiol groups, which bind to gold, to a gold surface. Then the complementary bases are similarly attached to the surface of a gold nanocluster via thiol linkages. The bases attached to the gold nanoclustres are then immobilized on the gold surface by spotting solutions. The immobilization is governed by the complementary base pairing. For the synthesis of the non-canonical basepair A:A, one needs to functionalize both the gold surface and gold cluster with Adenine. Hereafter, we denote the nonprotonated basepairs as $\mathrm{A}: \mathrm{T}_{N P}, \mathrm{G}: \mathrm{C}_{N P}, \mathrm{~A}: \mathrm{A}_{N P}$ and the protonated basepairs as $\mathrm{A}: \mathrm{T}_{P}, \mathrm{G}: \mathrm{C}_{P}, \mathrm{~A}: \mathrm{A}_{P}$.

Upon optimization of the nonprotonated and the protonated basepairs, we find that the basepairs do not get aligned perpendicular to the surface of the gold cluster. The tilt with respect to the surface is about $106^{\circ}$ and this is due to the presence of the thiol linker which are used to attach the basepairs to the gold cluster surface. In Fig.1(b), (c) and (d), we present the optimized geometries of $\mathrm{A}: \mathrm{T}_{N P, P}, \mathrm{G}: \mathrm{C}_{N P, P}$ and $\mathrm{A}: \mathrm{A}_{N P, P}$, respectively. We find that the $\mathrm{A}: \mathrm{T}_{N P}, \mathrm{G}: \mathrm{C}_{N P}$ and $\mathrm{A}: \mathrm{A}_{N P}$ form planar structures due to almost linear H-bonding. However, the planarity of the $\mathrm{H}$-bonds are lost on protonation in $\mathrm{A}: \mathrm{T}_{P}, \mathrm{G}: \mathrm{C}_{P}$ and $\mathrm{A}: \mathrm{A}_{P}$.

For A: $\mathrm{T}_{P}$ (Fig.1(b)), we find that the protonation leads to the formation of a bidentate $\mathrm{H}-$ bond between two $\mathrm{N}-\mathrm{H}$ and one $\mathrm{O}\left(1.82 \AA, 145^{\circ}\right.$ and $\left.1.92 \AA, 141^{\circ}\right)$ as compared to the linear N-H...O $\left(1.72 \AA, 173^{\circ}\right)$ and N...H-N $(1.67 \AA$, $\left.178^{\circ}\right) \mathrm{H}$-bonds in $\mathrm{A}: \mathrm{T}_{N P}$. For G: $\mathrm{C}_{P}$ (Fig.1(c)), the protonation disrupts the central N-H...N H-bond and leads to the reorientation of the bases to form two N-H...O (2.02 $\AA, 174^{\circ}$ and $\left.1.67 \AA, 157^{\circ}\right)$ H-bonds as compared to the two N-H...O $\left(1.86 \AA\right.$ A $179^{\circ}$ and $\left.1.62 \AA, 179^{\circ}\right)$ H-bonds and one N-H..N $\left(1.84 \AA\right.$ A $\left.178^{\circ}\right) \mathrm{H}$-bond in G: $\mathrm{C}_{N P}$. The case of $\mathrm{A}: \mathrm{A}_{P}$ (Fig.1(d)) is distinct. The protonation in this case disrupts the two N-H...N H-bonds and the resultant structure is stabilized by partial $\pi-\pi$ interactions between the Adenine bases. In $\mathrm{A}: \mathrm{A}_{N P}$, there are two equivalent N-H...N $\left(1.84 \AA 2,178^{\circ}\right)$ H-bonds. Thus, the main point of our findings is that the protonation affects the nature and the strength of the H-bonds which leads to a considerable change in the structure of the basepairs with little change in energy as the $\mathrm{N}-\mathrm{H}$... $\mathrm{N} \mathrm{H}$-bond has the lowest H-Bond energy. We investigate the transport phenomenon of the non-protonated and the protonated basepairs to analyze the role played by $\mathrm{H}$-bonds and the $\pi-\pi$ interactions affecting the electronic structure of the basepairs thereby the related conductivity characteristics.

To understand the transport properties of the systems under study, we have calculated the transmission function $\mathrm{T}(\mathrm{E})$ at different bias voltages using the Green's function methodology 14. The Hamiltonian $(\mathrm{H})$ and the overlap (S) matrices are obtained from the self-consistent DFT calculations. To model the effect of bulk electrodes, we modify the Hamiltonian of the system by adding the imaginary self energy terms, $\Sigma_{L, R}$, (L and R being left and right electrode indices respectively) arising due to the bulk gold, to the orbitals of the gold cluster. Thus, the original Hamiltonian $\mathrm{H}$ gets modified to $\bar{H}$ as

$$
\bar{H}=H+\Sigma_{L}+\Sigma_{R}
$$

With the modified Hamiltonian, we calculate the Green's function $\mathrm{G}(\mathrm{E})$ and the transmission $\mathrm{T}(\mathrm{E})$ is then calculated as

$$
T(E)=\operatorname{Tr}\left[\Gamma_{L} G(E) \Gamma_{R} G^{+}(E)\right]
$$

where $\Gamma_{L, R}$ are the anti hermetian parts of the self-energy matrices which describe the broadening of the energy lev- 

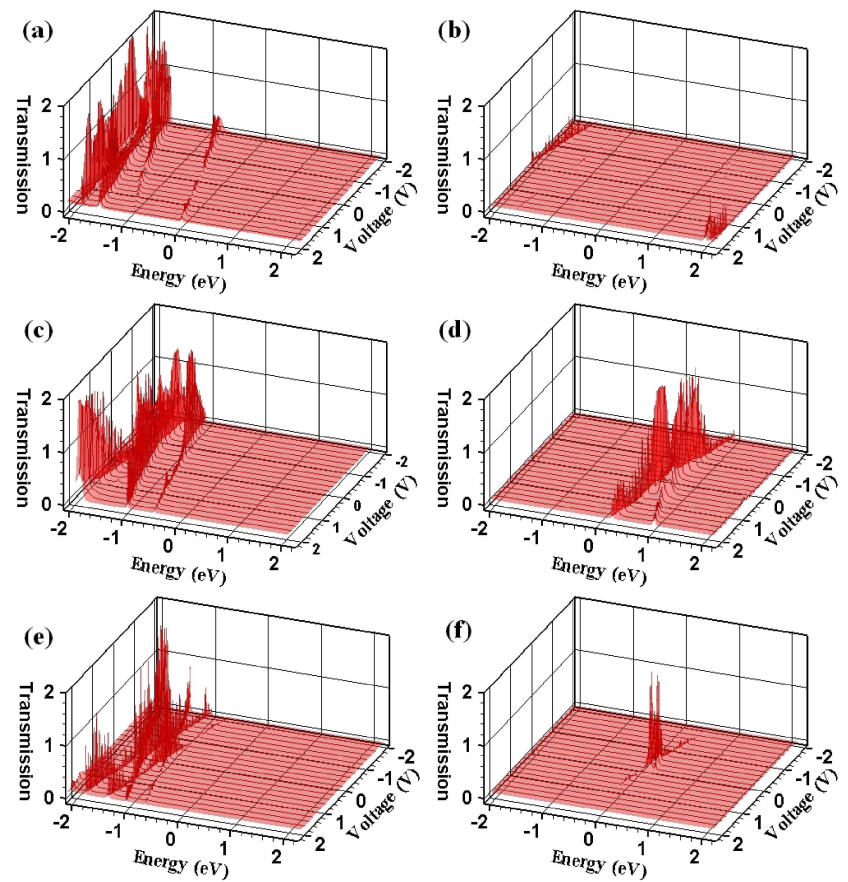

FIG. 2: (Color online) Transmission $\mathrm{T}(\mathrm{E})$ as function of applied bias voltage. (a) $\mathrm{G}: \mathrm{C}_{N P}$, (b) $\mathrm{G}: \mathrm{C}_{P}$, (c) $\mathrm{A}: \mathrm{T}_{N P}$, (d) $\mathrm{A}: \mathrm{T}_{P},(\mathrm{e}) \mathrm{A}: \mathrm{A}_{N P}$ and (f) $\mathrm{A}: \mathrm{A}_{P}$.

els of the system due to the coupling to the electrodes.

$$
\Gamma_{L, R}(E)=i\left(\Sigma_{L, R}-\Sigma_{L, R}^{+}\right)
$$

In Fig.2, we plot the transmission $\mathrm{T}(\mathrm{E})$ as a function of bias voltage for $\mathrm{G}_{(N P, P)}, \mathrm{A}: \mathrm{T}_{(N P, P)}$ and $\mathrm{A}: \mathrm{A}_{(N P, P)}$. We restrict our discussion to the low-energy molecular orbitals which govern the transport phenomenon around the Fermi level $\left(E_{F}\right)$. Note that, since we have incorporated a large number of $\mathrm{Au}$ atoms to model the extended molecule, the relative location of the molecular levels with respect to $E_{F}$ is accurately estimated. On examining the $\mathrm{T}(\mathrm{E})$ for all the cases in the energy range $E_{F}-2 \mathrm{eV}$ to $E_{F}+2 \mathrm{eV}$, we find that the transmission for the non-protonated basepairs is governed by the occupied molecular orbitals whereas for the protonated basepairs, the transmission is controlled by the unoccupied molecular orbitals. We note that this is a direct consequence of the positive AEA (Adiabatic Electron Affinity) associated with the free bases 15 .

Before analyzing the $\mathrm{T}(\mathrm{E})$ data for the individual cases we point out the general features in the $\mathrm{T}(\mathrm{E})$ data plotted in Fig.2 . The naturally occurring Watson -Crick basepairs A:T and G:C have an inherent asymmetry due to which we find that the transmission peaks follow the changes in the chemical potential of one electrode. Whereas, for the symmetric basepair A:A, we find no such preference and the transmission peaks follow a symmetric trend in both the forward and reverse bias directions. Similar shifts of molecular levels under
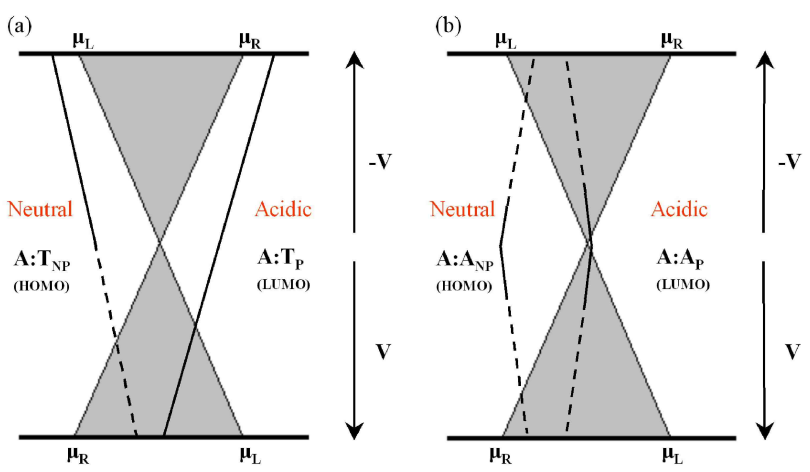

FIG. 3: (color online) (a) Rectification mechanism for $\mathrm{A}: \mathrm{T}_{P}$. (b) Switching action in noncanonical basepair A:A. Shaded portion illustrates the integrating region. Arrows indicate the bias direction. Solid lines indicate strong transmission and dotted line indicate weak transmission.

the influence of an external bias have been studied before for a symmetrically and asymmetrically contacted molecule [16].

The HOMO band of $\mathrm{G}_{\mathrm{C}} \mathrm{C}_{N P}$ (Fig.2(a)) which is 0.35 $\mathrm{eV}$ below the $E_{F}$ shows very weak zero bias transmission and it does not improve with a change in the bias voltage. However, the HOMO-1 band which is $1.35 \mathrm{eV}$ below the $E_{F}$, shows strong transmission in the negative bias region, but not in the forward bias region. Also we note that in the negative bias region, the transmission peak moves away from the $E_{F}$ and thus we do not expect this transmission band to enter the integration region and have any pronounced effect on the Current-Voltage (IV) characteristics of the system. For G: $\mathrm{C}_{P}$ (Fig.2(b)), we do not find any strong transmission peaks in the energy range considered by us. Thus, our calculations show that the $\mathrm{G}: \mathrm{C}_{N P}$ and $\mathrm{G}: \mathrm{C}_{P}$ do not posses any features of interest for molecular electronics applications.

However, the situation is very different for the A:T systems. As can be seen from Fig.2c, the HOMO band for $\mathrm{A}: \mathrm{T}_{N P}$, which is $0.75 \mathrm{eV}$ below the $E_{F}$, shows strong transmission in the negative bias region, but not in the forward bias region. However, due to the asymmetric nature of the electron distribution in the basepair, the transmission peak moves away from the $E_{F}$ with increase in bias, in the negative bias region. Thus, we do not expect this transmission band to enter the integration region to generate any nonzero I-V characteristics for small reverse bias. However, for the A: $\mathrm{T}_{P}$ (Fig.2(d)), we observe that the LUMO band which is $0.47 \mathrm{eV}$ above the $E_{F}$ shows very strong transmission at zero bias and appreciable transmission in the negative bias as well as in the forward bias region. We also find that in the positive bias region, the transmission peak appears right at the $E_{F}$.

From the above observations, we describe a rectification mechanism for the A:T basepair (Fig.3(a)). In the 
negative bias region, the $\mathrm{HOMO}$ level for $\mathrm{A}: \mathrm{T}_{N P}$ and the LUMO level for $\mathrm{A}: \mathrm{T}_{P}$ do not enter into the integrating region (illustrated by the shaded region in Fig.3(a)). However, in the positive bias region, low-energy levels, namely, HOMO level of A:T $T_{N P}$ and the LUMO level of $\mathrm{A}: \mathrm{T}_{P}$ enter the integration region and thus we would expect an increase in the current signal for both $\mathrm{A}: \mathrm{T}_{N P}$ and $\mathrm{A}: \mathrm{T}_{P}$ for this bias polarity. Interestingly however, due to the weak transmission (indicated by the dotted lines in Fig. 3a) associated with the HOMO level for $\mathrm{A}: \mathrm{T}_{N P}$ in the positive bias region, the resulting current would be quite small. Thus, we note that, only in the $\mathrm{A}: \mathrm{T}_{P}$ system, we will observe an asymmetric rectifying I-V profile with significant current in the positive bias polarity and negligible electron flow in the reverse polarity of applied bias.

On analyzing the $\mathrm{T}(\mathrm{E})$ data for the A:A systems, we find that using the reversible equilibrium between $\mathrm{A}: \mathrm{A}_{N P}$ and $\mathrm{A}: \mathrm{A}_{P}$, the $\mathrm{A}: \mathrm{A}$ basepair can be used as a molecular switch at zero bias. At equilibrium (zero bias), the HOMO level of A: $\mathrm{A}_{N P}$ (Fig.2(e)) is $1.05 \mathrm{eV}$ below the $E_{F}$, whereas the LUMO level of A:A $\mathrm{A}_{P}$ (Fig.2(f)) occurs right at the $E_{F}$. This is quite significant, as even at zero bias, if the $\mathrm{pH}$ of the system is changed from neutral to acidic, we would see a switching behavior which is reversible. From the $\mathrm{T}(\mathrm{E})$ data we also find that this switching action can be observed in a very small applied bias window as the transmission of the LUMO level of $\mathrm{A}: \mathrm{A}_{P}$ decreases rapidly under the influence of an external bias in both the directions. We depict this scenario schematically in Fig. 3(b). The mechanism of the switching behavior can be explained by examining the HOMO and LUMO orbitals of $\mathrm{A}: \mathrm{A}_{N P}$ and A:AP, respectively. The same are plotted in Fig.4. We find that the $\mathrm{HOMO}$ of $\mathrm{A}: \mathrm{A}_{N P}$ is not delocalized over the entire basepair, rather is localized on the individual Adenine bases, whereas, the LUMO of $\mathrm{A}: \mathrm{A}_{P}$ is delocalized over the entire basepair. Note that, such large delocalization characteristics of $\mathrm{A}: \mathrm{A}_{P}$ stems from the $\pi-\pi$ interactions between the Adenine bases. In a recent experimental study by Seferos et al. the strength of $\pi$ - $\pi$ coupling versus conjugated coupling was studied and it was found that the $\pi-\pi$ coupling improved the conduction due to strong through space electrostatic interactions. This is the same reason why $\mathrm{A}: \mathrm{A}_{P}$ shows stronger conducting profile in comparison to $\mathrm{A}: \mathrm{A}_{N P}$ [17, 18. Also, the fact that the switching interactions is controlled due to the interplay between weak interactions, namely, H-bonding and $\pi$ - $\pi$ interactions, promise technological applications with easy tunability. Our findings thus suggest that the noncanonical A:A systems are prospective candidates for switching action under very small $\mathrm{pH}$ change to the overall system.

In conclusion, we find that transport characteristics of the A:T and the G:C basepairs vary significantly from each other. The H-bonds in A:T are energetically very
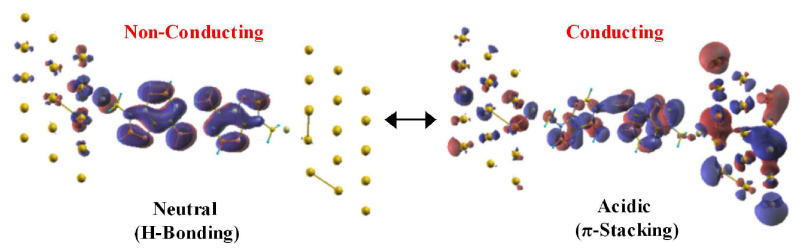

FIG. 4: (Color online) Orbital plots of the basepairs; HOMO of $\mathrm{A}: \mathrm{A}_{N P}$ (left) and LUMO of $\mathrm{A}: \mathrm{A}_{P}$ (right).

different from those in G:C; while A:T favors efficient transport, G:C does not. We find that under mild acidic conditions, the A:T basepairs can be used as efficient molecular rectifiers; the action of which can be enhanced by tuning the $\mathrm{pH}$ of the system. Also we find that the non-canonical A:A basepair can be used as an efficient molecular switch at zero bias, where the switching behavior is controlled by an interplay between weak intermolecular H-bonding and $\pi$ - $\pi$ interactions.

S. S. M. thanks CSIR for financial support. S. K. P. acknowledges the DST, Government of India for financial support.

[1] J. M. Tour, Molecular Electronics: Commercial Insights, Chemistry, Devices, Architecture and Programming, World Scientific: River Edge, NJ, 2003.

[2] (a) C. M. Niemeyer, Angew. Chem. Int. Ed. 40, 4128 (2001). (b) N. C. Seeman, Nature (London) 421, 427 (2003).

[3] (a) V. Bhalla, R. P. Bajpai, and L. M. Bharadwaj, EMBO reports 4, 442 (2003). (b) R. G. Endres, D. L. Cox, and R. R. P. Singh, Rev. Mod. Phys. 76, 195 (2004). (c) S. S. Mallajosyula and S. K. Pati, Phys. Rev. Lett. 98, 136601 (2007).

[4] W. Saenger, Principles of Nucleic Acid Structure (Springer, New York, 1984).

[5] G. M. Morales, et al., J. Am. Chem. Soc. 127, 10456 (2005).

[6] J. M. Soler, et al., J. Phys.: Condens. Matter 14, 2745 (2002).

[7] Gaussian 03, Revision C.02, M. J. Frisch, et al. Gaussian, Inc., Wallingford CT, 2004.

[8] J. P. Perdew, K. Burke and M. Ernzerhof, Phys. Rev. Lett. 77, 3865 (1996).

[9] O. F. Sankey and D. J. Niklewski, Phys. Rev. B 40, 3979 (1989).

[10] R. Di Felice, A. Selloni, E. Molinari, J. Phys. Chem. B. 107, 1151 (2003).

[11] (a) P. J. Hay and W. R. Wadt, J. Chem. Phys. 82, 270 (1985). (b) P. J. Hay and W. R. Wadt, J. Chem. Phys. 82, 299 (1985) (c) W. R. Wadt and P. J. Hay, J. Chem. Phys. 82, 284 (1985).

[12] J. E. Del Bene, J. Phys. Chem. 87, 367 (1983).

[13] (a) C. A. Mirkin, et al., Nature (London) 382, 607 (1996). (b) A. P. Alivisatos, et al., Nature (London) 382, 609 (1996).

[14] S. Datta, Electronic Transport in Mesoscopic Systems 
(Cambridge University Press, Cambridge, 1995).

[15] S. S. Mallajosyula, A. Datta, and S. K. Pati, Syn. Met. 155, 398 (2005).

[16] J. Taylor, M. Brandbyge, and K. Stokbro, Phys. Rev. Lett. 89, 138301 (2002).

[17] D. S. Seferos, et al., Proc. Natl. Acad. Sci. (USA) 102,
$8821(2005)$.

[18] (a) S. Lakshmi, A. Datta and S. K. Pati, Phys. Rev. B 72, 45131 (2005). (b) S. Lakshmi and S. K. Pati, Phys. Rev. B 72, 193410 (2005). 